\title{
REMARKS ON HALL $\pi$-SUBGROUPS OF A FINITE GROUP
}

\section{YONGCAI REN}

School of Mathematics

Sichuan University

Chengdu, 610064

P. R. China

e-mail: 18981711749@163.com

\begin{abstract}
In this paper, we discuss Hall $\pi$-subgroups of a finite group and generalize some results on Sylow $p$-subgroups.
\end{abstract}

In this paper, we discuss Hall $\pi$-subgroups of a finite group and generalize some results on Sylow $p$-subgroups.

Throughout this paper, the term group always means a finite group, and the letter $G$ always denotes a group. Our remaining notations are standard (see [1] and [2]). Some additional notations will be introduced as we go along.

2010 Mathematics Subject Classification: 20D15, 20 D20.

Keywords and phrases: finite group, $S_{\pi}$-subgroup, normal $\pi$-complement, focal subgroup.

Received June 26, 2019

(C) 2019 Scientific Advances Publishers 
Let $H$ be a subgroup of $G$. $H$ is called a Hall $\pi$-subgroup of $G$ provided $H$ is a $\pi$-group and $|G: H|$ is a $\pi^{\prime}$-number. In this paper, a Hall $\pi$-subgroup of $G$ is called an $S_{\pi}$-subgroup of $G$. When $\pi=\{p\}$, an $S_{\pi}$-subgroup of $G$ is simply a Sylow $p$-subgroup of $G$.

When $\pi=\{p\}$, by Sylow's theorem $G$ possesses an $S_{\pi}$-subgroup. But, for an arbitrary set of primes $\pi$, a group $G$ may or may not possess an $S_{\pi}$-subgroup.

Definition. Let $H$ be a subgroup of $G$, and let

$$
\operatorname{Foc}_{G}(H)=<h^{-1} h^{g} \mid g \in G, h, h^{g} \in H>.
$$

The subgroup $\mathrm{Foc}_{G}(H)$ is called the focal subgroup of $H$ with respect to G.

We shall use the following facts without explicit reference:

(i) $\mathrm{Foc}_{G}(H) \leq H \cap[H, G]$.

(ii) If $H \unlhd G$, then, $F_{o c}(H)=[H, G]$.

(iii) Let $N=N_{G}(H)$. Then, we have $F_{O} c_{N}(H)=[H, N]$.

(iv) Let $H$ and $K$ be subgroups of $G$ such that $H \subseteq K$. Suppose that two elements of $H$ are conjugate in $G$ if and only if they are conjugate in $K$. Then, we have $\operatorname{Foc}_{G}(H)=\operatorname{Foc}_{K}(H)$.

For convenient reference, we restate several known results as the following lemmas.

Lemma 1 ([3, (2.7), p. 141]). Let $H$ be a subgroup of $G$. The following two propositions hold:

(i) The focal subgroup $\mathrm{Foc}_{G}(H)$ contains the derived group $H^{\prime}$ of $H$. We have $\operatorname{Foc}_{G}(H) \unlhd H$, and the factor $H / F_{0 c}(H)$ is abelian. 
(ii) If a subgroup $K$ of $H$ contains the focal subgroup $F_{G o c}(H)$, then, $K \unlhd H$ and $H / K$ is abelian. Let $V$ be the transfer from $G$ into $H / K$. Then, for any $h \in H$, we have

$$
V(h)=h^{n} K,
$$

where $n=|G: H|$. If the order of $H / K$ is prime to $n$, then, $V$ is a surjection and we have $H \cap \operatorname{Ker} V=K$, where Ker $V$ denotes the kernel of the transfer homomorphism $V$.

Lemma 2 ([3, Theorem 3.2, p. 166]). If $G$ contains a nilpotent $S_{\pi}$-subgroup then, the following two statements are true:

(1) Any two $S_{\pi}$-subgroup of $G$ are conjugate in $G$.

(2) Any $\pi$-subgroup of $G$ is contained in an $S_{\pi}$-subgroup of $G$.

Lemma 3 ([3, Exercise (2), p. 186]). Suppose that $G$ contains a nilpotent $S_{\pi}$-subgroup and let $Q$ be a $\pi$-subgroup of $G$. Then, $N_{G}(Q)$ possesses a nilpotent $S_{\pi}$-subgroup.

Lemma 4 ([3, Theorem 3.7, p. 169]). Suppose that $G$ is $S_{\pi}$-separable. Then, the following three statements are true:

(1) G contains an $S_{\pi}$-subgroup.

(2) Any two $S_{\pi}$-subgroup of $G$ are conjugate in $G$.

(3) Any $\pi$-subgroup of $G$ is contained in an $S_{\pi}$-subgroup of $G$.

Lemma 5 ([3, (5.11), p. 108]). Suppose that $G$ contains an $S_{\pi}$-subgroup $H$ and let $N$ be a normal subgroup of $G$. Then, $H \cap N$ is an $S_{\pi}$-subgroup of $N$. 
Lemma 6 ([3, (2.29), p. 157]). Let $R, K$, and $N$ be subgroups of $G$ such that $N \unlhd G$ and $K \unlhd R$. Assume that the following three conditions are satisfied:

(1) $K=O^{\pi}(R \cap N)$.

(2) $(R \cap N) / K \subseteq \Phi(R / K)$.

(3) The index $|G: R|$ is a $\pi^{\prime}$-number.

Then, we have $K=R \cap O^{\pi}(N)$.

The following Lemma 7 is a part of [3, Theorem 2.9, p. 18].

Lemma 7. Let $G$ be a nilpotent group. If $T$ is a proper subgroup of $G$. Then, $T$ is a proper subgroup of $N_{G}(T)$.

Suppose that $G$ contains an $S_{\pi}$-subgroup $H$. We say that $G$ possesses a normal $\pi$-complement if there exists a normal subgroup $D$ of $G$ such that

$$
G=H D \text { and } H \cap D=\{1\}
$$

The normal subgroup $D$ is called a normal $\pi$-complement of $G$. It is clear that $D$ is a normal Hall $\pi^{\prime}$-subgroup of $G$.

As an immediate corollary of Lemma 1 we have the following:

Theorem 1. Suppose that $G$ contains an $S_{\pi}$-subgroup $H$. Then, there is a normal subgroup $D$ of $G$ such that

$$
G=H D \text { and } H \cap D=F_{O C}(H) .
$$

In particular, if the focal subgroup $\operatorname{Foc}_{G}(H)=\{1\}$, then, there is a normal subgroup $D$ of $G$ such that

$$
G=H D \text { and } H \cap D=\{1\},
$$

that is, G has a normal $\pi$-complement $D$. 
Proof. We have $F_{o c}(H) \unlhd H$ and $H / F_{o c}(H)$ is abelian (see Lemma 1). Let $V$ be the transfer from $G$ into $H / F_{o c}(H)$. Since $H$ is an $S_{\pi}$-subgroup of $G$, the order of $H / F_{0 c}(H)$ is prime to $|G: H|$. Then, by Lemma 1 we know that $V$ is a surjective homomorphism from $G$ onto $H / F_{G o c}(H)$ and $H \cap \operatorname{Ker} V=F_{O c}(H)$. It follows that

$$
G / \operatorname{Ker} V \cong H / F_{O c}(H) \text {. }
$$

Hence, we have

$$
|G|=\left|H / F_{O c}(H)\right| \cdot|\operatorname{Ker} V|=|H||\operatorname{Ker} V| /\left|F_{O o c}(H)\right| .
$$

We have $|H \cdot \operatorname{Ker} V|=|H||\operatorname{Ker} V| /|H \cap \operatorname{Ker} V|$. Then, since $H \cap \operatorname{Ker} V=$ $\operatorname{Foc}_{G}(H)$, we get

$$
|H \cdot \operatorname{Ker} V|=|H||\operatorname{Ker} V| /\left|\operatorname{Foc}_{G}(H)\right| .
$$

So, we have $|G|=|H \cdot \operatorname{Ker} V|$, and hence we have $G=H \cdot \operatorname{Ker} V$. Set $D=\operatorname{Ker} V$. Then, we have that $D \unlhd G, G=H D$ and $H \cap D=F_{O o c}(H)$. This completes the proof.

Theorem 2. Suppose that $G$ contains an $S_{\pi}$-subgroup $H$. If $G$ possesses a normal $\pi$-complement, then, the following three statements are true:

(i) For $h_{1}, h_{2} \in H$, if $h_{1}$ and $h_{2}$ are conjugate in $G$, then, $h_{1}$ and $h_{2}$ are conjugate in $H$.

(ii) $\operatorname{Foc}_{G}(H)=H^{\prime}$.

(iii) For every $\pi$-subgroup $Q$ of $G, N_{G}(Q) / C_{G}(Q)$ is a $\pi$-group.

Proof. By hypothesis, there is a normal subgroup $D$ of $G$ such that

$$
G=H D \text { and } H \cap D=\{1\}
$$

Furthermore, $D$ is a normal Hall $\pi^{\prime}$-subgroup of $G$. 
Suppose that $h^{g} \in H$ with $h \in H$ and $g \in G$. We have $g=x d$, where $x \in H$ and $d \in D$. Then, $h^{g}=h^{x d}=d^{-1} h^{x} d=h^{x}\left[h^{x}, d\right]$. It follows that $\left[h^{x}, d\right]=\left(h^{x}\right)^{-1} h^{g} \in H$. On the other hand, since $d \in D$ and $D \unlhd G$, we have $\left[h^{x}, d\right] \in D$. Hence, we have $\left[h^{x}, d\right] \in H \cap D=\{1\}$. Then, we get $h^{g}=h^{x}$. This implies that statement (i) is true.

Statement (ii) is an immediate consequence of statement (i).

Let $Q$ be any $\pi$-subgroup of $G$. Since $D$ is a normal Hall $\pi^{\prime}$-subgroup of $G, D$ contains all $\pi^{\prime}$-elements of $G$. Let $x$ be any $\pi^{\prime}$-element in $N_{G}(Q)$. Then, we have $x \in D$ and $[Q, x] \leq Q \cap D$. Since $D$ is a $\pi^{\prime}$-group and $Q$ is a $\pi$-group, we have $Q \cap D=\{1\}$. Hence, we have $[Q, x]=\{1\}$. This implies that $N_{G}(Q) / C_{G}(Q)$ is a $\pi$-group. So, statement (iii) is true. This completes the proof.

If every nontrivial factor group of $G$ is not an abelian $\pi$-group, that is, if $G / K$ is not an abelian $\pi$-group for every proper normal subgroups $K$ of $G$, then, we set $G^{\prime}(\pi)=G$. We assume that $G$ possesses a nontrivial abelian $\pi$-factor group. Then, $G^{\prime}(\pi)$ is the smallest normal subgroup of $G$ such that the factor group is a nontrivial abelian $\pi$-group. Clearly, $G^{\prime}(\pi)$ is a characteristic subgroup of $G$.

Theorem 3. The following four propositions hold:

(1) $G^{\prime}(\pi)=G^{\prime} O^{\pi}(G)$, where $G^{\prime}$ is the derived group of $G$.

(2) Suppose that $G$ contains an $S_{\pi}$-subgroup $H$. Then, $G=H \cdot G^{\prime}(\pi)$ $=H \cdot O^{\pi}(G)$.

(3) Suppose that $G$ contains an $S_{\pi}$-subgroup H. Then, $G^{\prime}(\pi)=G$ if and only if $\mathrm{Foc}_{G}(H)=H$.

(4) $O^{\pi}(G)=O^{\pi}\left(G^{\prime}(\pi)\right)$. 
Proof. We leave the verifications of (1) and (2) to the reader.

The verification of (3): By hypothesis, $H$ is an $S_{\pi}$-subgroup of $G$.

Suppose that $G^{\prime}(\pi)=G$. There is a normal subgroup $D$ of $G$ such that $G / D \cong H / F_{G}(H)$ (see the proof of Theorem 1). If $F_{o c}(H) \neq H$, then, $H / F_{0 c}(H)$ is a nontrivial abelian $\pi$-group (see Lemma 1), and so $G / D$ is a nontrivial abelian $\pi$-group. This implies that $G^{\prime}(\pi) \neq G$, a contradiction. So, we have Foc $_{G}(H)=H$.

Conversely, suppose that $\operatorname{Foc}_{G}(H)=H$. Since $H$ is an $S_{\pi}$-subgroup of $G$, by (2) we have $G=H \cdot G^{\prime}(\pi)$. It is obvious that we have

$$
\operatorname{Foc}_{G}(H) \subseteq H \cap G^{\prime} \subseteq H \cap G^{\prime}(\pi) .
$$

Then, since $F_{0 c}(H)=H$, we get $H \subseteq H \cap G^{\prime}(\pi)$, and so we have $H \subseteq G^{\prime}(\pi)$. Then, by (2) we have $G=H \cdot G^{\prime}(\pi)=G^{\prime}(\pi)$.

The verification of (4): Set $K=O^{\pi}\left(G^{\prime}(\pi)\right)$. Then, $\left.G^{\prime}(\pi)\right) / K$ is a $\pi$-group and $K \unlhd G$. Since both $G / G^{\prime}(\pi)$ and $G^{\prime}(\pi) / K$ are $\pi$-groups, $G / K$ is a $\pi$-group. It follows that $O^{\pi}(G) \subseteq K \subseteq G^{\prime}(\pi)$, and so we have $O^{\pi}(G) \subseteq G^{\prime}(\pi)$. Since $G / O^{\pi}(G)$ is a $\pi$-group, the subgroup $G^{\prime}(\pi) / O^{\pi}(G)$ of $G / O^{\pi}(G)$ is a $\pi$-group, and so we have $O^{\pi}\left(G^{\prime}(\pi)\right) \subseteq$ $O^{\pi}(G)$, that is, $K \subseteq O^{\pi}(G)$. Then, we have $O^{\pi}(G) \subseteq K \subseteq O^{\pi}(G)$, and thus we have $O^{\pi}(G)=K$, that is, $O^{\pi}(G)=O^{\pi}\left(G^{\prime}(\pi)\right)$. This completed the proof of the theorem.

Theorem 4. Suppose that $G$ contains an $S_{\pi}$-subgroup H. Then, we have

$$
\operatorname{Foc}_{G}(H)=H \cap G^{\prime}=H \cap G^{\prime}(\pi),
$$

where $G^{\prime}$ is the derived group of G. Furthermore, we have

$$
H / F_{O C}(H) \cong G / G^{\prime}(\pi) .
$$


Proof. Since $\operatorname{Foc}_{G}(H) \subseteq H \cap G^{\prime} \subseteq H \cap G^{\prime}(\pi)$, by Theorem 3(3) we may assume that $F_{0 c}(H) \neq H$. By Lemma 1, the $\pi$-group $H / F_{o c}(H)$ is abelian. Let $V$ be the transfer from $G$ into $H / F_{o c}(H)$. By Lemma 1, we have

$$
H \cap \operatorname{Ker} V=F_{O c}(H), \text { and } G / \operatorname{Ker} V \cong H / F_{O o c}(H) .
$$

It follows that $G / \operatorname{Ker} V$ is an abelian $\pi$-group. This implies that $G^{\prime}(\pi) \subseteq \operatorname{Ker} V$. Then, we have

$$
\operatorname{Foc}_{G}(H) \subseteq H \cap G^{\prime} \subseteq H \cap G^{\prime}(\pi) \subseteq H \cap \operatorname{Ker} V=F_{o c}(H) .
$$

It follows that

$$
\operatorname{Foc}_{G}(H)=H \cap G^{\prime}=H \cap G^{\prime}(\pi)
$$

and

$$
H / F_{G o c}(H)=H /\left(H \cap G^{\prime}(\pi)\right) \cong H G^{\prime}(\pi) / G^{\prime}(\pi) .
$$

Then, since $G=H \cdot G^{\prime}(\pi)$ by Theorem 3(2), we get

$$
H / F_{o c}(H) \cong G / G^{\prime}(\pi) .
$$

This completes the proof.

Theorem 5. The following propositions hold:

(1) Suppose that $G$ contains an $S_{\pi}$-subgroup $H$ and that $H$ is nilpotent. For $x, y \in Z(H)$, if $x$ and $y$ are conjugate in $G$, then, $x$ and $y$ are conjugate in $N_{G}(H)$.

(2) Suppose that $G$ is $\pi$-separable, and let $H$ be an $S_{\pi}$-subgroup of $G$. For $x, y \in Z(H)$, if $x$ and $y$ are conjugate in $G$, then, $x$ and $y$ are conjugate in $N_{G}(H)$. (By Lemma 4, a $\pi$-separable group possesses an $S_{\pi}$-subgroup.) 
Proof. The proof of (1): By assumption, there exists an element $g \in G$ such that $y=x^{g}$. It follow that $C_{G}(y)=\left(C_{G}(x)\right)^{g}$.

Since $x, y \in Z(H)$, we have that $H \leq C_{G}(x)$ and $H \leq C_{G}(y)$. Then, we have $H^{g} \leq\left(C_{G}(x)\right)^{g}=C_{G}(y)$. It follows that both $H \leq C_{G}(y)$ and $H^{g} \leq C_{G}(y)$. Then, since $H$ is a nilpotent $S_{\pi}$-subgroup of $G$ by hypothesis, $H$ and $H^{g}$ are nilpotent $S_{\pi}$-subgroups of $C_{G}(y)$. Hence, by Lemma 2, there exists an element $u \in C_{G}(y)$ such that $H^{u}=H^{g}$. So, we get $H=H^{g u^{-1}}$, and thus we have $g u^{-1} \in N_{G}(H)$. Since $y=x^{g}$ and $u \in C_{G}(y)$, we have $y=y^{u^{-1}}=x^{g u^{-1}}$. Then, since $g u^{-1} \in N_{G}(H), x$ and $y$ are conjugate in $N_{G}(H)$.

By using Lemma 4 and the same arguments as in the proof of (1), we can prove that (2) holds. This completes the proof of the theorem.

Theorem 6. Suppose that $G$ contains an $S_{\pi}$-subgroup $H$ and that $H$ is abelian. Set $N=N_{G}(H)$. The following propositions hold:

(1) For $x, y \in H$, if $x$ and $y$ are conjugate in $G$, then, $x$ and $y$ are conjugate in $N$.

(2) $\operatorname{Foc}_{G}(H)=\mathrm{Foc}_{N}(H)=[H, N]$.

(3) $H \cap G^{\prime}=[H, N]=H \cap N^{\prime}$.

(4) $H=(H \cap Z(N)) \times[H, N]$.

(5) $H \cap G^{\prime} \cap Z(G)=\{1\}$.

(6) Either $G^{\prime} \cap Z(G)=\{1\}$ or $G^{\prime} \cap Z(G)$ is a $\pi^{\prime}$-group.

(7) $G / G^{\prime}(\pi) \cong H \cap Z(N) \cong N / N^{\prime}(\pi)$. 
(8) $G / O^{\pi}(G) \cong H \cap Z(N) \cong N / O^{\pi}(N)$.

(9) $O^{\pi}(G)=G \Leftrightarrow H \cap Z(N)=\{1\} \Leftrightarrow O^{\pi}(N)=N$.

(10) $N_{G}(H)=C_{G}(H) \Leftrightarrow \operatorname{Foc}_{G}(H)=\{1\}$.

(11) If $H \unlhd G$ and $O^{\pi}(G)=G$, then, there exists a subgroup $D$ of $G$ such that $G=H D, H \cap D=\{1\}$ and $N_{G}(D)=D$.

Proof. (i) By hypothesis, the $S_{\pi}$-subgroup $H$ is abelian. Hence, by Theorem 5(1), we conclude that proposition (1) holds.

(ii) By proposition (1), we have $F_{0 c}(H)=F_{0 c}(H)$. Since $H \unlhd N=$ $N_{G}(H)$, we have $F_{o c}(H)=[H, N]$. So, proposition (2) holds.

(iii) By Theorem 4, we have that $F_{o c}(H)=H \cap G^{\prime}$ and $F_{o c}(H)=$ $H \cap N^{\prime}$, and so by proposition (2), we conclude that proposition (3) holds.

(iv) By Shure-Zassenhaus theorem (see [4, Theorem 2.1, p. 221]), we have $N=N_{G}(H)=H Q$, where $Q$ is a Hall $\pi^{\prime}$-subgroup of $N$. It is clear that $Q$ acts on $H$ by conjugation. Then, since $(|H|,|Q|)=1$, we have $H=C_{H}(Q) \times[H, Q]$ (see [5, p. 156 and p. 239], or see [3, p. 7]). Since $N=H Q$ and $H$ is abelian, we have that $C_{H}(Q)=H \cap Z(N)$ and $[H, N]=[H, H Q]=[H, Q]$ (see $[3,(1.1)$, p. 3]). So, we have $H=(H \cap Z(N)) \times[H, N]$, that is, proposition (4) holds.

(v) Proposition (5) is an immediate consequence of propositions (3) and (4).

(vi) Proposition (6) is an immediate consequence of proposition (5) and Lemma 5.

(vii) By Theorem 4, we have $G / G^{\prime}(\pi) \cong H / F_{0 c}(H)$, and so by propositions (2) and (4), we get $G / G^{\prime}(\pi) \cong H \cap Z(N)$. By using the same arguments, we get $N / N^{\prime}(\pi) \cong H \cap Z(N)$. So, proposition (7) holds. 
(viii) We have $G=H O^{\pi}(G)$ (see Theorem 3(2)), and so we have $G / O^{\pi}(G) \cong H /\left(H \cap O^{\pi}(G)\right.$. Hence, since $H$ is an abelian $\pi$-group, $G / O^{\pi}(G)$ is an abelian $\pi$-group. It follows that $G^{\prime}(\pi) \subseteq O^{\pi}(G)$. Then, since $G^{\prime}(\pi)=G^{\prime} O^{\pi}(G)$ (see Theorem $3(1)$ ), we get $G^{\prime}(\pi)=O^{\pi}(G)$. By exactly the same arguments, we conclude that $N^{\prime}(\pi)=O^{\pi}(N)$. So, by proposition (7), we conclude that proposition (8) holds.

(ix) Proposition (9) is an immediately consequence of proposition (8).

(x) Proposition (10) is an immediate corollary of propositions (2) and (4).

(xi) We now prove proposition (11). By hypothesis, $H \unlhd G$ and $O^{\pi}(G)=G$. Since $H$ is a normal $S_{\pi}$-subgroup of $G$, by Schur-Zassenhaus theorem there is a subgroup $D$ of $G$ such that $G=H D$ and $H \cap D=\{1\}$. Since $H \unlhd G$, we have $N=N_{G}(H)=G$. Since $O^{\pi}(G)=G$, by proposition (8) we have $H \cap Z(G)=H \cap Z(N)=\{1\}$. It is clear that $\left[N_{H}(D), D\right] \leq H \cap D=\{1\}$. Then, since $H$ is abelian and $G=H D$, we have $N_{H}(D) \leq Z(G)$, and so we have $N_{H}(D) \leq H \cap Z(G)=\{1\}$. Thus, since $G=H D$, we conclude that $N_{G}(D)=D$, completing the proof of proposition (11). This completes the proof of the theorem.

Note: Suppose that $G$ contains an $S_{\pi}$-subgroup. Then, $H \cap G^{\prime} \cap$ $Z(G) \leq H^{\prime}$ (see [6, Theorem 10.8, p. 235]). This result contains Theorem $6(5)$.

Corollary 1. Suppose that $G$ contains an abelian $S_{\pi}$-subgroup $H$. Then, the following four statements are equivalent:

(i) G possesses a normal $\pi$-complement.

(ii) $F_{O o c}(H)=\{1\}$.

(iii) No two distinct elements of $H$ are conjugate in $G$.

(iv) $N_{G}(H)=C_{G}(H)$. 
Proof. (i) $\Rightarrow$ (ii): See Theorem 2 .

(ii) $\Leftrightarrow$ (iii): This is obvious.

(ii) $\Leftrightarrow$ (iv): See Theorem 6(10).

(ii) $\Rightarrow$ (i): See Theorem 1 .

Theorem 7. Suppose that $O^{\pi}(G)$ possesses an abelian $S_{\pi}$-subgroup $H$. Then, $G$ splits over $O^{\pi}(G)$.

Proof. Set $K=O^{\pi}(G), T=N_{G}(H)$ and $N=N_{K}(H)$.

By hypothesis, $H$ is an abelian $S_{\pi}$-subgroup of $K$. Then, $H$ is a normal $S_{\pi}$-subgroup of $N=N_{K}(H)$, and so by Schur-Zassenhaus theorem there exists an $S_{\pi^{\prime}}$-subgroup $D$ of $N$ such that

$$
N=H D \text { and } H \cap D=\{1\} .
$$

Clearly, $N_{H}(D) \leq H \cap Z(N)$. Since $O^{\pi}\left(O^{\pi}(G)\right)=O^{\pi}(G)$, we have $O^{\pi}(K)=K$. Then, by Theorem 6(9) we have $H \cap Z(N)=\{1\}$, and so we get $N_{H}(D)=\{1\}$.

Since $K=O^{\pi}(G) \unlhd G$, we have $N \unlhd T$. Let $x$ be any element of $T$. We have $D^{x} \leq N$. Then, by Schur-Zassenhaus theorem (see [4, Theorem 2.1, p. 221]), there is an element $y \in N$ such that $D^{x}=D^{y}$. This implies that $x y^{-1} \in N_{T}(D)$. So, we get

$$
T=N \cdot N_{T}(D)=H D N_{T}(D)
$$

It is obvious that $N=T \cap K=T \cap O^{\pi}(G)$. Then, we have $T K / K \cong T /(T \cap K)=T / N$. Hence, since $G / K=G / O^{\pi}(G)$ is a $\pi$-group, $T / N$ is a $\pi$-group. So, we conclude that $D$ is a Hall $\pi^{\prime}$-subgroup 
of $T$. Then, $D$ is a normal Hall $\pi^{\prime}$-subgroup of $N_{T}(D)$, and so by SchurZassenhaus theorem we have $N_{T}(D)=H_{1} D=D H_{1}$, where $H_{1}$ is an $S_{\pi}$-subgroup of $N_{T}(D)$. It follows that

$$
T=H D N_{T}(D)=H D D H_{1}=H D H_{1}=N H_{1}=H_{1} N .
$$

Since $H$ is a normal $S_{\pi}$-subgroup of $N$ and $H_{1}$ is a $\pi$-group, we have $H_{1} \cap N \leq H$, and so we have $H_{1} \cap N=H_{1} \cap H$.

Since $H_{1} \leq N_{T}(D)$, we have $H_{1} \cap H \leq N_{H}(D)$. Then, since $N_{H}(D)=\{1\}$, we have $H_{1} \cap H=\{1\}$.

Let $x$ be any element of $G$. Then, $H^{x}$ is an $S_{\pi}$-subgroup of $K=O^{\pi}(G)$. By Lemma 2, there is an element $y \in K$ such that $H^{x y}=H$. This implies that $x y \in N_{G}(H)=T$. So, we conclude that $G=T K=T O^{\pi}(G)$. It follows that

$$
G=H_{1} N K=H_{1} K=H_{1} O^{\pi}(G) .
$$

We have

$$
H_{1} \cap O^{\pi}(G)=H_{1} \cap K \subseteq T \cap K=N
$$

and so we have

$$
H_{1} \cap O^{\pi}(G)=H_{1} \cap K \cap N=H_{1} \cap N=H_{1} \cap H=\{1\} .
$$

Then, we conclude that $G=H_{1} O^{\pi}(G)$ and $H_{1} \cap O^{\pi}(G)=\{1\}$, that is, $G$ splits over $O^{\pi}(G)$. This completes the proof.

The lower $\pi$-series of $G$ is the following sequence of characteristic subgroups of $G$ :

$$
\{1\} \subseteq O_{\pi^{\prime}}(G) \subseteq O_{\pi^{\prime}, \pi}(G) \subseteq O_{\pi^{\prime}, \pi, \pi^{\prime}}(G) \subseteq \cdots
$$


If $G$ is $\pi$-separable, then, the lower $\pi$-series of $G$ terminates in $G$ (see [4, Theorem 3.1, p. 227]). For a $\pi$-separable group $G$, the $\pi$-length of $G$ is the number of $\pi$-facters of the lower $\pi$-series of $G$.

Theorem 8. Let $G$ be $\pi$-separable and $H$ be an $S_{\pi}$-subgroup of $G$. The following three propositions hold:

(1) $C_{G}\left(H \cap O_{\pi^{\prime}, \pi}(G)\right) \subseteq O_{\pi^{\prime}, \pi}(G)$.

(2) If $H$ is abelian, then, either $G=O_{\pi^{\prime}, \pi}(G)$ or $G=O_{\pi^{\prime}, \pi, \pi^{\prime}}(G)$.

(3) If $H$ is abelian, then, the $\pi$-length of $G$ is 1 .

Proof. The proof of (1): Set $\bar{G}=G / O_{\pi^{\prime}}(G)$. Since $G$ is $\pi$-separable, we have

$$
C_{\bar{G}}\left(O_{\pi}(\bar{G})\right) \subseteq O_{\pi}(\bar{G})
$$

(see [4, Theorem 3.2, p. 228]).

Set $R=H \cap O_{\pi^{\prime}, \pi}(G)$. By Lemma $5, R$ is an $S_{\pi}$-subgroup of $O_{\pi^{\prime}, \pi}(G)$, and so $\bar{R}=O_{\pi}(\bar{G})$. Then, setting $T=C_{G}(R)$, we have $\bar{T} \subseteq C_{\bar{G}}\left(O_{\pi}(\bar{G})\right)$ and consequently, we have $C_{G}(R)=T \subseteq O_{\pi^{\prime}, \pi}(G)$ by $(*)$.

The proof of (2): By hypothesis $H$ is abelian, and so by (1) we get $H \subseteq O_{\pi^{\prime}, \pi}(G)$. Then, since $H$ is an $S_{\pi}$-subgroup of $G, G / O_{\pi^{\prime}, \pi}(G)$ is a $\pi^{\prime}$-group, and so either $G=O_{\pi^{\prime}, \pi}(G)$ or $G=O_{\pi^{\prime}, \pi, \pi^{\prime}}(G)$.

(3) is an immediate consequence of (2), completing the proof of the theorem.

A group $G$ is said to be $\pi$-nilpotent if $G$ has a normal Hall $\pi^{\prime}$-subgroup. In particular, if $G$ is either a $\pi$-group or a $\pi^{\prime}$-group, then, $G$ is $\pi$-nilpotent. 
Suppose that $G$ has a normal Hall $\pi^{\prime}$-subgroup $D$. Then, for any subgroup $K$ of $G, K \cap D$ is a normal Hall $\pi^{\prime}$-subgroup of $K$. If $N$ is a normal subgroup of $G$, then, $D N / N$ is a normal Hall $\pi^{\prime}$-subgroup of $G / N$ (see [3, (2.28), p. 156]). Therefore, we conclude that subgroups of a $\pi$-nilpotent group are $\pi$-nilpotent and factor groups of a $\pi$-nilpotent group are $\pi$-nilpotent.

Suppose that $G$ has a normal Hall $\pi^{\prime}$-subgroup $D$. Then, by SchurZassenhuas theorem, there is a subgroup $H$ of $G$ such that

$$
G=H D \text { and } H \cap D=\{1\} .
$$

It is clear that $H$ is an $S_{\pi}$-subgroup of $G$. So, $D$ is a normal $\pi$-complement of $G$. Hence, we conclude that if $G$ is $\pi$-nilpotent, then, $G$ has a normal $\pi$-complement. Conversely, if $G$ has a normal $\pi$-complement, then, it is obvious that $G$ is $\pi$-nilpotent.

It is easy to see that $G$ is $\pi$-nilpotent if and only if $O^{\pi}(G)$ is a $\pi^{\prime}$-group.

If $G$ has a normal Hall $\pi^{\prime}$-subgroup $D$, then, it is obvious that $D$ is a characteristic subgroup of $G$.

Theorem 9. Suppose that $G$ contains a nilpotent $S_{\pi}$-subgroup $H$. Let $K$ be a subgroup of $G$. Assume that $K$ satisfies the following two conditions:

(1) $H \cap K$ is an $S_{\pi}$-subgroup of $K$.

(2) Any two elements of $H \cap K$ which are conjugate in $K$ are conjugate in $H$. Then, $K$ is $\pi$-nilpotent. 
Proof. To prove that $K$ is $\pi$-nilpotent, we need only to show that $O^{\pi}(K)$ is a $\pi^{\prime}$-group. Set $T=H \cap O^{\pi}(K)$. We have $T=(H \cap K) \cap O^{\pi}(K)$, and so by condition (1) and Lemma 5 we conclude that $T$ is an $S_{\pi}$-subgroup of $O^{\pi}(K)$. Therefore, in order to show that $O^{\pi}(K)$ is a $\pi^{\prime}$-group, we need only to show that $T=\{1\}$.

Suppose that $T \neq\{1\}$. Set $D=O^{\pi}(K)$. Condition (2) implies that $F_{o c} D(T) \subseteq F_{0 c_{H}}(T)$. Then, since $F_{O c}(T) \subseteq T \cap[T, H]$, we have

$$
\operatorname{Foc}_{D}(T) \subseteq T \cap[T, H]
$$

Suppose that $T=T \cap[T, H]$. Then, we have $T \subseteq[T, H]$. This implies that $T \subseteq[T, H, H], T \subseteq[T, H, H, H]$, and so on. So, since $T \subseteq H$, we conclude that, for any positive integer $n$, we have $T \subseteq C_{n}(H)$, where $C_{n}(H)$ denotes the $n$-th term of the lower central series of $H$. By hypothesis, $H$ is nilpotent, and so there exists a positive integer $n$ such that $C_{n}(H)=\{1\}$. It follows that $T=\{1\}$, a contradiction. Hence, we have $T \neq T \cap[T, H]$, that is, $T \cap[T, H]$ is a proper subgroup of $T$. Then, by $(* *)$, we conclude that the focal subgroup $\operatorname{Foc}_{D}(T)$ is a proper subgroup of $T$. Then, by Theorem 3 we have $O^{\pi}(D) \neq D$. However, we have $O^{\pi}\left(O^{\pi}(K)\right)=O^{\pi}(K)$, that is $O^{\pi}(D)=D$, a contradiction. So, we conclude that $T=\{1\}$. This completes the proof.

Theorem 10. Suppose that $G$ contains a nilpotent $S_{\pi}$-subgroup $H$. Let $R$ be a subgroup of $G$ such that $H \subseteq R$. If $R^{\prime}(\pi)=R \cap G^{\prime}(\pi)$, then, $O^{\pi}(R)=R \cap O^{\pi}(G)$. 
Proof. By hypothesis, $H$ is a nilpotent $S_{\pi}$-subgroup of $R$. Set $K=O^{\pi}\left(R^{\prime}(\pi)\right)$. By Theorem 3(4) we have $K=O^{\pi}(R)$. Then, by Theorem 3(2) we have $R=H K$. Since $H$ is nilpotent, $R / K=H K / K$ is nilpotent, and so $\Phi(R / K)$ contains the derived group $(R / K)^{\prime}$ of $R / K$ (see [7, 3.10 Satz, p. 271]).

By Theorem 3(1), we have $R^{\prime}(\pi)=R^{\prime} O^{\pi}(R)=R^{\prime} K$, and thus we have

$$
(R / K)^{\prime}=R^{\prime} K / K=R^{\prime}(\pi) / K,
$$

that is, the derived group of $R / K$ is $R^{\prime}(\pi) / K$.

To sum up, we have

$$
R^{\prime}(\pi) / K \subseteq \Phi(R / K)
$$

Since $H$ is an $S_{\pi}$-subgroup of $G$ and $H \subseteq R$, the index $[G: R]$ is a $\pi^{\prime}$-number. Since we have $R^{\prime}(\pi)=R \cap G^{\prime}(\pi)$ by hypothesis, we get that $K=O^{\pi}\left(R \cap G^{\prime}(\pi)\right)$ and that $\left(R \cap G^{\prime}(\pi)\right) / K \subseteq \Phi(R / K)$. It follows that $R, K$, and $G^{\prime}(\pi)$ satisfy all the conditions of Lemma 6 . So, by Lemma 6 , we have $K=R \cap O^{\pi}\left(G^{\prime}(\pi)\right)$. By Theorem 3(4), we have $O^{\pi}\left(G^{\prime}(\pi)\right)=O^{\pi}(G)$, and hence we have $O^{\pi}(R)=K=R \cap O^{\pi}(G)$. This completes the proof.

Corollary 2. Suppose that $G$ contains a nilpotent $S_{\pi}$-subgroup $H$. Then, the following three statements are equivalent:

(1) $G$ is $\pi$-nilpotent.

(2) Any two elements of $H$ which are conjugate in $G$ are conjugate in $H$.

(3) $\mathrm{Foc}_{G}(H)=H^{\prime}$. 
Proof. (1) $\Rightarrow$ (2): See Theorem 2.

$(2) \Rightarrow(3)$ : It is obvious.

(3) $\Rightarrow$ (1): By Theorem 4, we have

$$
\operatorname{Foc}_{G}(H)=H \cap G^{\prime}=H \cap G^{\prime}(\pi) .
$$

On the other hand, we have $F_{o c}(H)=H^{\prime}$ by hypothesis, and so we get

$$
H^{\prime}=H \cap G^{\prime}=H \cap G^{\prime}(\pi) .
$$

Since $H$ is a $\pi$-group, we have $O^{\pi}(H)=\{1\}$, and so by Theorem 3(1), we get $H^{\prime}(\pi)=H^{\prime} \cdot O^{\pi}(H)=H^{\prime}$. It follows that

$$
H^{\prime}(\pi)=H \cap G^{\prime}(\pi)
$$

Then, by Theorem 10, we have

$$
O^{\pi}(H)=H \cap O^{\pi}(G)
$$

Then, since $O^{\pi}(H)=\{1\}$, we obtain that $H \cap O^{\pi}(G)=\{1\}$. Hence, since $G=H \cdot O^{\pi}(G)$ by Theorem 3(2), $G$ has a normal $\pi$-complement, and this implies that $G$ is $\pi$-nilpotent. This completes the proof of the corollary.

Corollary 3. Suppose that $G$ contains a nilpotent $S_{\pi}$-subgroup $H$. If there is a normal subgroup $D$ of $G$ such that $G=H D$ and $H \cap D=H^{\prime}$, then, $G$ is $\pi$-nilpotent.

Proof. By hypothesis we have

$$
G / D \cong H / H^{\prime}
$$

Then, since $H / H^{\prime}$ is an abelian $\pi$-group, we have $G^{\prime}(\pi) \subseteq D$, and so we have $\left|G / G^{\prime}(\pi)\right| \geq|G / D|=\left|H / H^{\prime}\right|$. Hence, by Theorem 4 we get $|H / F o c(H)| \geq\left|H / H^{\prime}\right|$. This implies that $|F o c(H)| \leq\left|H^{\prime}\right|$. Then, since $H^{\prime} \subseteq F_{o c}(H)$ (see Lemma 1), we get $F_{O c}(H)=H^{\prime}$. So, by Corollary 2, $G$ is $\pi$-nilpotent. This completes the proof. 
For the implication (1) $\Leftrightarrow$ (2) in Corollary 2, also see [6, Crollary 10.41, p. 260]. Theorem 9 contains the implication (2) $\Rightarrow(1)$ in Corollary 2. By Lemma 2 and a theorem of Dade (see [3, (2.4), p. 277]), we also obtain the implication (2) $\Rightarrow(1)$ in Corollary 2. But, the proof of the theorem of Dade uses representation theory.

By Lemma 4 and the theorem of Dade, the following Theorem 11 holds.

Theorem 11. Suppose that $G$ is $\pi$-separable, and let $H$ be an $S_{\pi}$-subgroup of $G$. Assume that any two elements of $H$ which are conjugate in $G$ are conjugate in H. Then, $G$ is $\pi$-nilpotent.

Theorem 12. Suppose that $G$ contains an $S_{\pi}$-subgroup $H$, and let $Q$ be a normal subgroup of $H$. If $C_{H}(Q) \subseteq Q$, then,

(1) $Z(Q)=1$ and $C_{G}(Q)$ is a $\pi^{\prime}$-group, or

(2) there is a $\pi^{\prime}$-subgroup D such that

$$
C_{G}(Q)=Z(Q) \times D
$$

Furthermore, we have $D=O_{\pi^{\prime}}\left(N_{G}(Q)\right)$.

Proof. By hypothesis we have $C_{H}(Q) \subseteq Q$. This implies that $C_{H}(Q)=Z(Q)$.

By hypothesis, we have $H \subseteq N_{G}(Q)$. Then, since $H$ is an $S_{\pi}$-subgroup of $G, H$ is an $S_{\pi}$-subgroup of $N_{G}(Q)$. So, since $C_{G}(Q)$ $\unlhd N_{G}(Q), C_{H}(Q)$ is an $S_{\pi}$-subgroup of $C_{G}(Q)$ by Lemma 5 . Then, since $C_{H}(Q)=Z(Q), Z(Q)$ is an $S_{\pi}$-subgroup of $C_{G}(Q)$. Hence, if $Z(Q)=\{1\}$, then, $C_{G}(Q)$ is a $\pi^{\prime}$-group. Now we assume that $Z(Q) \neq\{1\}$. Clearly, we have $Z(Q) \subseteq Z\left(C_{G}(Q)\right)$. It follows from Shur-Zaddenhaus theorem that there is a $\pi^{\prime}$-subgroup $D$ of $G$ such that

$$
C_{G}(Q)=Z(Q) \times D
$$


Clearly, $D$ is a normal Hall $\pi^{\prime}$-subgroup of $C_{G}(Q)$. Then, Dchar $C_{G}(Q) \unlhd N_{G}(Q)$ and $D \subseteq O_{\pi^{\prime}}\left(N_{G}(Q)\right)$. On the other hand, since $Q$ is a $\pi$-group, we have

$$
\left[Q, O_{\pi^{\prime}}\left(N_{G}(Q)\right)\right] \subseteq Q \cap O_{\pi^{\prime}}\left(N_{G}(Q)\right)=\{1\} .
$$

So, $O_{\pi^{\prime}}\left(N_{G}(Q)\right) \subseteq C_{G}(Q)$ and we get $D=O_{\pi^{\prime}}\left(N_{G}(Q)\right)$. This completes the proof.

Theorem 13. Suppose that $G$ is $\pi$-separable, and let $H$ be an $S_{\pi}$-subgroup of $G$. Let $T$ be a subgroup of $H$. Then, there is a subgroup $U$ of $H$ such that

(1) $N_{H}(U)$ is an $S_{\pi}$-subgroup of $N_{G}(U)$, and

(2) $U$ is conjugate to $T$ in $G$.

Proof. Since $G$ is $\pi$-separable, subgroups of $G$ are $\pi$-separable. So, by Lemma 4, we know that $N_{H}(T)$ is contained in an $S_{\pi}$-subgroup $S$ of $N_{G}(T)$, and the subgroup $S$ is contained in an $S_{\pi}$-subgroup $Q$ of $G$.

We have $S=Q \cap N_{G}(T)$. By Lemma 4 , we have $H=Q^{x}$ for some element $x$ of $G$. Set $U=T^{x}$. Then, $T \subseteq S \subseteq Q$ and

$$
U=T^{x} \subseteq Q^{x}=H
$$

Furthermore, $S^{x}$ is an $S_{\pi}$-subgroup of $N_{G}(U)=N_{G}(T)^{x}$, and we have

$$
S^{x}=Q^{x} \cap N_{G}(T)^{x}=H \cap N_{G}(U)=N_{H}(U) .
$$

This completes the proof.

Theorem 14. Suppose that $G$ contains a nilpotent $S_{\pi}$-subgroup $H$. Then, for any subgroup $T$ of $H$, there is a subgroup $U$ of $H$ such that $U$ is conjugate to $T$ in $G$, and for the sequence of subgroups $U_{0}, U_{1}, \ldots$, defined by $U_{0}=U$ and inductively by $U_{i}=N_{H}\left(U_{i-1}\right)$, the subgroup $U_{i+1}$ is an $S_{\pi}$-subgroup of $N_{G}\left(U_{i}\right)$ for all $i=0,1 \ldots$. 
Proof. By hypothesis and Lemma $3, N_{G}(T)$ possesses a nilpotent $S_{\pi}$-subgroup. Let $V_{1}$ be a nilpotent $S_{\pi}$-subgroup of $N_{G}(T)$ which contains $T$ (see Lemma 2). For $i=1,2, \ldots$, by Lemma 3 we know that $N_{G}\left(V_{i}\right)$ possesses a nilpotent $S_{\pi}$-subgroup, and let $V_{i+1}$ be an $S_{\pi}$-subgroup of $N_{G}\left(V_{i}\right)$ which contains $V_{i}$ (see Lemma 2). By Lemma 7, $V_{i}$ is a proper subgroup of $V_{i+1}$, unless $V_{i}$ is an $S_{\pi}$-subgroup of $G$. So, the sequence

$$
T \subset V_{1} \subset V_{2} \subset \cdots
$$

will reach an $S_{\pi}$-subgroup $S$ of $G$ in a finite number of steps. By Lemma 2 , there is an element $x \in G$ such that $H=S^{x}$. Set $T^{x}=U$. Then, $V_{1}^{x}$ is an $S_{\pi}$-subgroup of $N_{G}(U)$, and

$$
V_{1}^{x}=H \cap N_{G}(U)=N_{H}(U) .
$$

For each $i, V_{i+1}^{x}$ is an $S_{\pi}$-subgroup of $N_{G}\left(V_{i}^{x}\right)$, and

$$
V_{i+1}^{x}=H \cap N_{G}\left(V_{i}^{x}\right)=N_{H}\left(V_{i}^{x}\right)
$$

Set $U_{i}=V_{i}^{x}$ for $i=1,2, \ldots$. Then, $U_{1}$ is an $S_{\pi}$-subgroup of $N_{G}(U)$, and

$$
U_{1}=H \cap N_{G}(U)=N_{H}(U) .
$$

For each $i, U_{i+1}$ is an $S_{\pi}$-subgroup of $N_{G}\left(U_{i}\right)$, and

$$
U_{i+1}=H \cap N_{G}\left(U_{i}\right)=N_{H}\left(U_{i}\right) .
$$

This completes the proof.

As an immediate corollary of Lemma 2 and Theorem 14, we have the following: 
Corollary 4. Suppose that $G$ contains a nilpotent $S_{\pi}$-subgroup and let $T$ be a $\pi$-subgroup of $G$. Then, there is an $S_{\pi}$-subgroup $H$ of $G$ such that $T \leq H$ and $N_{H}(T)$ is an $S_{\pi}$-subgroup of $N_{G}(T)$.

Theorem 15. Suppose that $G$ contains a nilpotent $S_{\pi}$-subgroup. Assume that $N_{G}(Q) / C_{G}(Q)$ is a $\pi$-group for every $S_{\pi}$-subgroup $Q$ of $G$. Then, for any two $S_{\pi}$-subgroup $H$ and $H^{\#}$ of $G$ and any $x \in H \cap H^{\#}$, there is an element $y \in C_{G}(x)$ such that $H^{\#}=H^{y}$.

Proof. Suppose by way of contradiction that the theorem does not hold. We choose the $S_{\pi}$-subgroups $H$ and $H^{\#}$ of $G$ such that the assertion of the theorem does not hold for $H$ and $H^{\#}$ and $\left|H \cap H^{\#}\right|$ is maximal. Set $T=H \cap H^{\#}$. Clearly, we have $H^{\#} \neq H$, and hence we have $T<H$. Then, since $H$ is nilpotent, by Lemma 7 we have $T<N_{H}(T)$.

By Corollary 4, there is an $S_{\pi}$-subgroup $H_{1}$ of $G$ such that $T \leq H_{1}$ and $N_{H_{1}}(T)$ is an $S_{\pi}$-subgroup of $N_{G}(T)$. Then, since $N_{G}(T) / C_{G}(T)$ is a $\pi$-group by hypothesis, we have $N_{G}(T)=C_{G}(T) \cdot N_{H_{1}}(T)$. Hence, by Lemma 2, we conclude that there is an element $y \in C_{G}(T)$ such that $\left(N_{G}(T)\right)^{y} \leq N_{H_{1}}(T)$. It follows that $T \leq N_{H^{y}}(T)$ and $N_{H^{y}}(T) \leq N_{H_{1}}(T)$. Then, we have $N_{H^{y}}(T) \leq H^{y} \cap H_{1}$. It follows that $\left|H \cap H^{\#}\right|=|T|$ $<\left|N_{H}(T)\right|=\left|\left(N_{H}(T)\right)^{y}\right|=\left|N_{H^{y}}(T)\right| \leq\left|H^{y} \cap H_{1}\right|$. Hence, we have $\left|H \cap H^{\#}\right|<\left|H^{y} \cap H_{1}\right|$. Then, by the choice of $H$ and $H^{\#}$, we see that the assertion of the theorem holds for $H^{y}$ and $H_{1}$. Then, noticing that $x \in T \leq H^{y} \cap H_{1}$, there is an element $z \in C_{G}(x)$ such that $H^{y z}=\left(H^{y}\right)^{z}=H_{1}$. Since $x \in T$ and $y \in C_{G}(T)$, we have $y \in C_{G}(x)$. So, we have $y z \in C_{G}(x)$. Set $w=y z$. Then, $w \in C_{G}(x)$ and $H^{w}=H_{1}$. 
Also, we have $T<H^{\#}$. So, by the above arguments, we conclude that there is an element $v \in C_{G}(x)$ such that $\left(H^{\#}\right)^{v}=H_{1}$. It follows that $w v^{-1} \in C_{G}(x)$ and $H^{w v^{-1}}=H^{\#}$. This is contrary to the choice of $H$ and $H^{\#}$. This contradiction proves the theorem.

Theorem 16. Suppose that $G$ contains a nilpotent $S_{\pi}$-subgroup. Then, $G$ is $\pi$-nilpotent if and only if $N_{G}(Q) / C_{G}(Q)$ is a $\pi$-group for every $\pi$-subgroup $Q$ of $G$.

Proof. $\Rightarrow$ : See Theorem 2.

$\Leftarrow$ : Suppose that $N_{G}(Q) / C_{G}(Q)$ is a $\pi$-group for every $\pi$-subgroup $Q$ of $G$.

Let $H$ be an $S_{\pi}$-subgroup of $G$, and let $N=N_{G}(H)$. Since $H \unlhd N$, we have $F_{O c}(H)=[H, N]$. Clearly, $H$ is an $S_{\pi}$-subgroup of $N$. Then, since $N_{G}(H) / C_{G}(H)$ is a $\pi$-group by our assumption, we have $N=N_{G}(H)=H \cdot C_{G}(H)$. It follows that

$$
\operatorname{Foc}_{N}(H)=[H, N]=\left[H, H \cdot C_{G}(H)\right]=[H, H]=H^{\prime} .
$$

Let $x \in H, g \in G$ and $x^{g} \in H$. Then, $x \in H \cap H^{g^{-1}}$, and therefore, by Theorem 15, we have

$$
H^{g^{-1}}=H^{y},
$$

for some $y \in C_{G}(x)$. Then, $y g \in N_{G}(H)=N$ and $x^{g}=x^{y g}$. Hence $x$ and $x^{g}$ are conjugate in $N=N_{G}(H)$. So, we conclude that $F_{o c}(H)=$ $F_{o c}(H)$. Then, since $F_{o c}(H)=H^{\prime}$, we get $F_{O c}(H)=H^{\prime}$, and so by Corollary 2 we know that $G$ is $\pi$-nilpotent. This completes the proof of the theorem. 


\section{References}

[1] H. E. Rose, A Course on Finite Groups, Springer-Verlag London Limited, 2009.

[2] B. Huppert, Character Theory of Finite Groups, Berlin: Walter der Gruyter, 1998.

[3] Michio Suzuki, Group Theory II, Springer, 1986.

[4] D. Gorensten, Finite Groups, Harper and Row, 1968.

[5] Michio Suzuki, Group Theory I, Springer, 1982.

[6] J. S. Rose, A Course on Group Theory, Cambridge University Press, 1978.

[7] B. Huppet, Endlich Gruppen I, Springer, 1967. 